\title{
Concomitância de Fibromialgia em Pacientes Portadores de Hipotireoidismo e de Alterações Tireoideanas em Pacientes com Fibromialgia
}

\section{Fibromyalgia Concomitance in Patients with Hypothyroidism and Thyroid Disorders in Patients with Fibromyalgia}

\author{
Marlene Freire $^{(1)}$, Reginaldo B. Teodoro ${ }^{(2)}$, Lilia Beatriz Oliveira ${ }^{(3)}$, Selma Freire C. Cunha ${ }^{(4)}$, \\ Beatriz P. Ferreira ${ }^{(5)}$, Maria de Fátima Borges ${ }^{(6)}$
}

\section{RESUMO}

Introdução: a fibromialgia (FM) pode ser definida como uma síndrome dolorosa musculoesquelética não-inflamatória de caráter crônico, tendo como sintomas mais comuns a fadiga e o sono não-reparador. Tais manifestações podem não resultar da FM, mas de outras doenças associadas, como o hipotireoidismo. Objetivo: descrever a ocorrência de FM em pacientes com hipotireoidismo, e os casos de hipotireoidismo em pacientes com FM, além de determinar os sintomas associados a essas condições clínicas. Métodos: foram avaliados 166 pacientes portadores de FM e 56 com hipotireoidismo. Os voluntários responderam a um questionário para detecção dos principais sinais e sintomas dessas doenças em estudo e foram submetidos às dosagens séricas de hormônio estimulador da tireóide (TSH), tiroxina livre (T4 livre) e anticorpo antitireoperoxidase (a-TPO). Resultados: nos 166 pacientes com FM, foram diagnosticados hipotireoidismo clínico ou subclínico em 35 (21,1\%). Dos 56 pacientes portadores de hipotireoidismo, em 36 (64,3\%) foi confirmado o diagnóstico de FM pelos critérios do American College of Rheumatology (ACR). Nos pacientes com hipotireoidismo previamente diagnosticado, a fadiga, o sono não-restaurador e a cefaléia crônica foram estatisticamente mais freqüentes naqueles que apresentavam diagnóstico de FM. A rigidez matinal foi mais prevalente nos pacientes com hipotireoidismo sem FM. Conclusão: há que se considerar a presença de manifestações clínicas ou laboratoriais de disfunção tireoideana nos pacientes portadores de FM e de FM em pacientes portadores de hipotireoidismo, para uma melhor abordagem diagnóstica e posterior tratamento dos mesmos.

Palavras-chave: fibromialgia, hipotireoidismo, tireoidite crônica auto-imune.

\begin{abstract}
Introduction: Fibromyalgia can be defined as a non-inflammatory musculoskeletal pain syndrome. It is a chronic condition, commonly associated with fatigue and non-restorative sleep. However, such symptoms may result from coexisting diseases, for instance hypothyroidism. Objective: Based on this evidence, the aim of this study was to describe the concomitance of fibromyalgia in patients with hypothyroidism, the cases of hypothyroidism in patients diagnosed with fibromyalgia and determine the symptoms associated with above clinical conditions. Methods: One hundred and sixty-six patients with fibromyalgia and 56 patients with hypothyroidism were evaluated. Volunteer patients answered a questionnaire to detect the main signs and symptoms of these diseases and then underwent serum determination of thyroid stimulating hormone (TSH), free thyroxine (FT4) and anti-thyroperoxidase (a-TPO). Results: Among 166 patients with fibromyalgia, 35 (21.1\%) were diagnosed with clinical or subclinical hypothyroidism. Among 56 patients with hypothyroidism, $36(64.3 \%)$ had the diagnosis of fibromyalgia confirmed, according to the American College of Rheumatology's (ACR) criteria. In patients with previously diagnosed hypothyroidism, fatigue, non-restorative sleep and chronic headache were symptoms statistically more frequent among those with the diagnosis of fibromyalgia. Morning stiffness prevailed in patients diagnosed with hypothyroidism and without fibromyalgia. Conclusion: We can concluded from this study that, in order to enhance the diagnostic approach and follow-up treatment, the presence of clinical or laboratorial manifestations of thyroid dysfunction in patients with fibromyalgia must be considered, as well as manifestations of fibromyalgia in patients with hypothyroidism.
\end{abstract}

Keywords: fibromyalgia, hypothyroidism, chronic autoimmune thyroiditis

\footnotetext{
Disciplina de Reumatologia da Faculdade de Medicina do Triângulo Mineiro (FMTM). Recebido em 22/03/05. Aprovado, após revisão, em 09/1 1/05.

1. Professora adjunta da Disciplina de Reumatologia da FMTM. 2. Médico assistente da Disciplina de Reumatologia da FMTM.

3. Médica residente da Disciplina de Reumatologia da FMTM.

4. Professora adjunta da Disciplina de Nutrologia da FMTM.

5. Professora assistente da Disciplina de Endocrinologia da FMTM.

6. Professora adjunta da Disciplina de Endocrinologia da FMTM

Endereço para correspondência: Marlene Freire, Faculdade de Medicina do Triângulo Mineiro, Departamento de Clínica Médica, Rua Getúlio Guaritá s/n, Bairro
}

Abadia, Uberaba, CEP 38025-440, MG, Brasil, telefone: (34) 3318-5229, fax: (34) 3332-7510, e-mail: m.freire@terra.com.br 


\section{INTRODUÇÃO}

A fibromialgia (FM) é definida como uma síndrome dolorosa musculoesquelética não-inflamatória de caráter crônico $^{(1)}$. A descrição das principais manifestações clínicas, como queixas álgicas distribuídas difusamente pelo corpo, sensação de fadiga e distúrbios do sono, foi feita por Sir Willian Gowers, em 1904, que introduziu o termo fibrosite. Em 1981, Yunus a denominou FM. Aos poucos, esta síndrome foi despertando interesse na prática clínica, em virtude de sua multissistemicidade e do elevado número de pacientes acometidos ${ }^{(1,2)}$. Vários estudos foram conduzidos com o objetivo de documentar as alterações que a envolvem $^{(1)}$. Em 1990, após estudo multicêntrico envolvendo 16 serviços de FM do Canadá e dos Estados Unidos, foram publicados os critérios de classificação de acordo com o American College of Rheumatology (ACR) que são: dor difusa persistente por mais de três meses e presença de dor em 11 dos 18 pontos dolorosos (tender points) $)^{(1,2,3)}$. Esta síndrome ocorre na proporção aproximada de nove mulheres para cada homem, na faixa etária de 30 a 50 anos, em pessoas com tendência à depressão, perfeccionistas $\mathrm{e}$ detalhistas. $^{(1,2)}$

A fadiga e o sono não-reparador são os sintomas mais comuns, embora, muitos outros tenham sido descritos ${ }^{(1,2,3)}$. Algumas das manifestações clínicas podem não se relacionar à FM, mas a outras doenças associadas. Entre elas, o hipotireoidismo tem despertado grande interesse pela similaridade de sintomas com a FM ou pela expressiva associação entre as condições clínicas ${ }^{(3-8)}$.

Baseados nessas evidências, propomos o presente estudo com os objetivos de descrever os casos de FM em pacientes com hipotireoidismo e de hipotireoidismo em pacientes com diagnóstico de FM, além de determinar os sintomas associados a essas condições clínicas.

\section{PACIENTES E MÉTODOS}

Foram avaliados 222 pacientes, sendo 166 portadores de FM, segundo os critérios diagnósticos do ACR selecionados no ambulatório de reumatologia da Faculdade de Medicina do Triângulo Mineiro (FMTM); 56 pacientes estavam em tratamento e acompanhamento regular no ambulatório de endocrinologia, com diagnóstico de hipotireoidismo. Todos os participantes responderam a um questionário para detecção dos principais sinais e sintomas das doenças em estudo. Os pacientes foram submetidos às dosagens séricas do hormônio estimulador da tireóide (TSH), tiroxina livre (T4 livre) e anticorpo antitireoperoxidase (a-TPO), utilizando-se o método de quimiluminescência e um sistema semi-automatizado do tipo IMMULLITE. Para tal, empregaram-se estojos comerciais da DPC (Diagnostic Products Corporation - Los Angeles - Estados Unidos).

A função tireoideana foi considerada normal quando as concentrações séricas estavam dentro dos valores de referência: TSH: $0,38-4,5 \mathrm{mUI} / \mathrm{mL}$; T4 livre: $0,8-2,3$ $\mathrm{ng} / \mathrm{dL}$ e a-TPO $<10 \mathrm{UI} / \mathrm{mL}$. O hipotireoidismo clínico foi diagnosticado nos pacientes que apresentaram elevação do TSH e diminuição dos valores de T4 livre; foi considerado subclínico quando a única alteração foi a elevação das concentrações de TSH. Concentrações elevadas de a-TPO foram consideradas como indicativos de tireoidite crônica auto-imune.

Os dados foram analisados no programa Statistica 6.0. Utilizou-se o teste t de Student para comparação de variáveis numéricas; a comparação entre proporções foi feita pelo teste exato de Fisher. Diferenças entre as variáveis foram significativas quando $\mathrm{p}<0,05$.

\section{RESULTADOS}

Entre os 166 pacientes portadores de FM, $162(97,6 \%)$ eram do sexo feminino. A média de idade foi de 49,5 \pm 10 anos, $140(84,3 \%)$ foram classificados como sendo de cor branca e $124(74,7 \%)$ eram casados. Vinte e oito (16,9\%) eram analfabetos e $30(18,1 \%)$ estiveram ou estavam afastados do trabalho em razão da sintomatologia dolorosa.

Dos 56 pacientes portadores de hipotireoidismo, 55 $(92,2 \%)$ eram do sexo feminino, com média de idade de $50,7 \pm 11$ anos, $49(87,5 \%)$ eram brancos, $38(67,9 \%)$ eram casados e 11 (19,6\%) estiveram ou estavam afastados do trabalho em razão da sintomatologia dolorosa. Não houve diferença estatística significativa entre os grupos quanto aos dados demográficos (Tabela 1).

Nos 56 pacientes com hipotireoidismo, $36(64,3 \%)$ apresentaram dor generalizada e 11 ou mais tender points, confirmando o diagnóstico de FM pelos critérios do ACR (Quadro 1).

Nos 166 pacientes portadores de FM, o hipotireoidismo clínico ou subclínico foi diagnosticado em 35 casos $(21,1 \%)$, sendo $16(45,7 \%)$ com hipotireoidismo subclínico por provável tireoidite crônica auto-imune (TSH elevado, T4 livre normal, a-TPO elevado), $10(28,6 \%)$ com hipotireoidismo subclínico idiopático (TSH discretamente elevado, T4 livre normal e a-TPO normal), 8 (22,9\%) com hipotireoidismo clínico por tireoidite crônica auto-imune (TSH elevado, T4 
livre diminuído e a-TPO elevado) e l (2,9\%) com hipotireoidismo por causa não definida (TSH elevado, T4 livre diminuído e a-TPO normal) (Quadro 2).

TABELA 1

DADOS DEMOGRÁFICOS DOS 222

PACIENTES PARTICIPANTES DA PESQUISA

\begin{tabular}{l|c|c|c}
\hline Variáveis & \multicolumn{2}{|c|}{ Pacientes } & \\
\hline & $\begin{array}{c}\text { Fibromialgia } \\
(\mathrm{n}=166)\end{array}$ & $\begin{array}{c}\text { Hipotireoidismo } \\
(\mathrm{n}=56)\end{array}$ & $\mathrm{p}=$ \\
\hline Sexo Feminino & $162(97,6 \%)$ & $55(92,2 \%)$ & 0,8 \\
\hline Idade (anos) & $49,5 \pm 10,1$ & $50,7 \pm 11,2$ & 0,4 \\
\hline Cor Branca & $140(84,3 \%)$ & $49(87,5 \%)$ & 0,6 \\
\hline Casados & $124(74,7 \%)$ & $38(67,9 \%)$ & 0,3 \\
\hline IMC (Kg/m $\left.{ }^{2}\right)$ & $25,9 \pm 4,8$ & $27 \pm 5,3$ & 0,1 \\
\hline Analfabetismo & $28(16,9 \%)$ & $4(7,1 \%)$ & 0,07 \\
\hline Absenteísmo & $30(18,1 \%)$ & $11(19,6 \%)$ & 0,5 \\
\hline
\end{tabular}

Quadro 1

Organograma DOS RESULTADOS DE PACIENTES COM HIPOTIREOIDISMO



Nos pacientes com hipotireoidismo previamente diagnosticado, a fadiga, o sono não-restaurador e a cefaléia crônica foram estatisticamente mais freqüentes naqueles em que foi feito o diagnóstico de FM. Por outro lado, a rigidez matinal foi mais prevalente nos pacientes com hipotireoidismo sem FM (Tabela 2).
QUADro 2

Organograma dos RESUltados DE PACIENTES COM FIBROMIALGIA

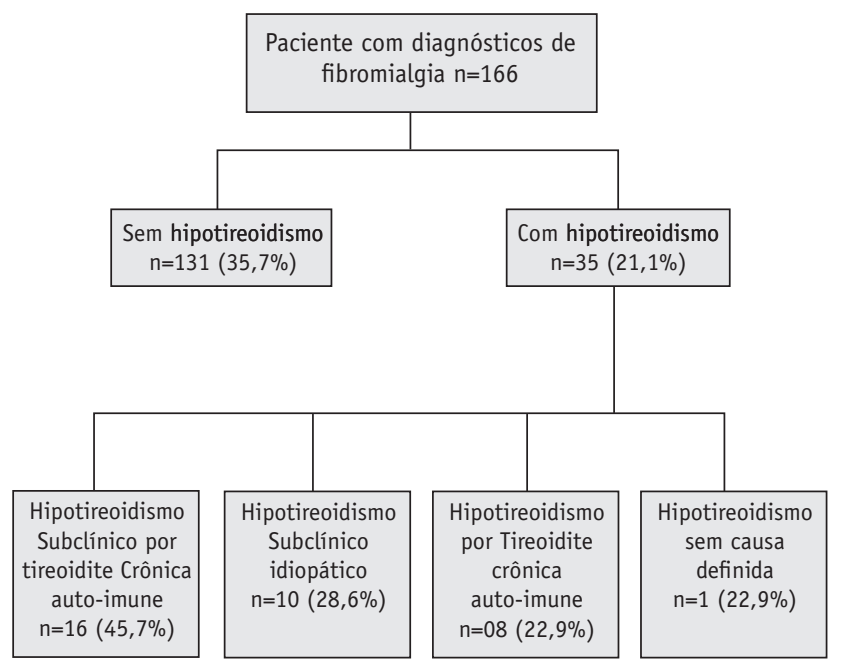

TABELA 2

Sintomas relacionados à Fibromialgia em pacientes COM HipotiReOIdismo PREVIAMENTE DIAGNOSTICADO

\begin{tabular}{|c|c|c|c|}
\hline \multirow[t]{2}{*}{ Sintomas } & \multicolumn{2}{|c|}{ Pacientes } & \multirow[b]{2}{*}{$p=$} \\
\hline & $\begin{array}{l}\text { Fibromialgia } \\
\qquad(n=20)\end{array}$ & $\begin{array}{c}\text { Hipotireoidismo } \\
+ \text { Fibromialgia } \\
(n=36)\end{array}$ & \\
\hline Ansiedade & $16(84,2 \%)$ & $33(91,7 \%)$ & 0,4 \\
\hline Fadiga & $7(36,8 \%)$ & $31(86,1 \%)$ & 0,0002 \\
\hline $\begin{array}{l}\text { Sono não- } \\
\text { restaurador }\end{array}$ & $7(36,8 \%)$ & $28(77,8 \%)$ & 0,003 \\
\hline Insônia & $8(42,1 \%)$ & $18(50 \%)$ & 0,6 \\
\hline Cefaléia crônica & $7(36,8 \%)$ & $24(66,7 \%)$ & 0,03 \\
\hline $\begin{array}{l}\text { Sensação de } \\
\text { edema }\end{array}$ & $8(42,1 \%)$ & $24(66,7 \%)$ & 0,08 \\
\hline Cólon irritável & $2(10,5 \%)$ & $6(1,67 \%)$ & 0,5 \\
\hline Parestesia & $11(57,9 \%)$ & $22(61,1 \%)$ & 0,8 \\
\hline Rigidez matinal & $14(73,7 \%)$ & $13(36,1 \%)$ & 0,008 \\
\hline
\end{tabular}

Não houve diferença estatística quanto à presença de sintomas relacionados ao hipotireoidismo quando os pacientes com FM e hipotireoidismo foram comparados com aqueles com FM sem hipotireoidismo (Tabela 3). 
TABELA 3

Sintomas Relacionados aO Hipotireoidismo EM PACIENTES COM Fibromialgia

\begin{tabular}{|c|c|c|c|}
\hline \multirow[t]{2}{*}{ Sintomas } & \multicolumn{2}{|c|}{ Pacientes } & \multirow[b]{2}{*}{$p=$} \\
\hline & $\begin{array}{l}\text { Fibromialgia }+ \\
\text { Hipotireoidismo } \\
(n=20)\end{array}$ & $\begin{array}{l}\text { Fibromialgia } \\
\qquad(n=131)\end{array}$ & \\
\hline $\begin{array}{l}\text { Intolerância ao } \\
\text { frio }\end{array}$ & $11(37,9 \%)$ & $49(35,8 \%)$ & 0,82 \\
\hline Pele ressecada & $20(69,0 \%)$ & $82(59,8 \%)$ & 0,4 \\
\hline $\begin{array}{l}\text { Queda de } \\
\text { cabelos }\end{array}$ & $13(44,8 \%)$ & $61(44,5 \%)$ & 0,9 \\
\hline $\begin{array}{l}\text { Memória } \\
\text { alterada }\end{array}$ & $24(82,8 \%)$ & $93(67,9 \%)$ & 0,1 \\
\hline $\begin{array}{l}\text { Concentração } \\
\text { alterada }\end{array}$ & $19(65,5 \%)$ & $96(67,9 \%)$ & 0,8 \\
\hline $\begin{array}{l}\text { Constipação } \\
\text { intestinal }\end{array}$ & $12(41,4 \%)$ & $44(32,1 \%)$ & 0,3 \\
\hline $\begin{array}{l}\text { Alteração do } \\
\text { peso }\end{array}$ & $17(58,6 \%)$ & $78(56,9 \%)$ & 0,9 \\
\hline $\begin{array}{l}\text { Alteração ciclo } \\
\text { menstrual }\end{array}$ & $8(27,6 \%)$ & $34(25,6 \%)$ & 0,8 \\
\hline Cansaço & $26(89,7 \%)$ & $115(83,9 \%)$ & 0,4 \\
\hline Dispnéia & $20(69,0 \%)$ & $86(62,8 \%)$ & 0,5 \\
\hline $\begin{array}{l}\text { Crescimento do } \\
\text { pescoço }\end{array}$ & $25(86,2 \%)$ & $119(86,9 \%)$ & 0,9 \\
\hline Rouquidão & $8(27,6 \%)$ & $30(21,9 \%)$ & 0,5 \\
\hline
\end{tabular}

\section{DISCUSSÃO}

Há vários anos, têm sido descrito casos de pacientes portadores de hipotireoidismo clínico e subclínico que apresentam sintomas musculoesqueléticos de origem não estabelecida, como fraqueza muscular, dores articulares e $\mathrm{FM}^{(1,2,6,7,8,9)}$. A incidência de sintomas musculoesqueléticos no hipotireoidismo tem sido estimada de 30 a $80 \%(4,5,10)$. Em nosso estudo, 64,3\% dos pacientes portadores de hipotireoidismo apresentavam quadro de dor generalizada associada a pelo menos 11 tender points preenchendo os critérios do ACR para FM.

Até o momento, foram publicados poucos dados que relacionam as disfunções tireoideanas em pacientes com FM. No presente estudo, dentre os 166 indivíduos com diagnóstico prévio de FM, 35 (21,1\%) apresentaram concentrações elevadas de TSH indicando associação com hipotireoidismo. Neeck e Riedel(5), em 1992, mostraram alteração da função tireoideana em pacientes com FM, com uma menor secreção de TSH, T3 e T4 após estímulo com terapia de reposição hormonal (TRH), comparados com indivíduos controles. Forslind, Fredriksson e Nived ${ }^{(11)}$ avaliaram 25 pacientes na ocasião do diagnóstico de FM primária e após cinco anos. No início do estudo, apenas um paciente apresentava o diagnóstico de hipotireoidismo; passados cinco anos, mais cinco haviam desenvolvido a doença, elevando a incidência de hipotireoidismo para $24 \%$ neste grupo. Shiroky et al ${ }^{(12)}$ encontraram quatro casos de hipotireoidismo (12\%) em 34 pacientes com FM.

Em 38 pacientes com FM, Lowe ${ }^{(13)}$ diagnosticou quatro casos de hipotireoidismo clínico (10,5\%) e em 20 $(52,6 \%)$ os testes de função tireoideana foram compatíveis com hipotireoidismo central. Destes, 11 pacientes $(28,9 \%)$ apresentavam respostas discretas de TSH frente ao estímulo com TRH, sugerindo hipotireoidismo hipofisário. Ferraccioli et a ${ }^{(14)}$ documentaram respostas insuficientes do TSH ao TRH em 5 de 24 pacientes com FM (20,8\%). De forma semelhante, Neeck e Riedel ${ }^{(5)}$ observaram baixa resposta de TSH em $100 \%$ dos seus 13 pacientes.

No que diz respeito à etiopatogênese da FM, ainda não existem estudos mostrando o exato mecanismo causador da doença ${ }^{(1)}$. A hipótese é que os baixos níveis de serotonina observados no soro $^{(15,16)}$ e líquido cerebroespinhal ${ }^{(17,18)}$, sejam os responsáveis pelas manifestações clínicas. Tal hipótese não tem sido comprovada, em virtude de resultados pouco expressivos com o uso terapêutico de antidepressivos tricíclicos e inibidores seletivos de recaptação de serotonina (ISRS $^{(19,20)}$, quando comparados com placebo. Tais dados sugerem a existência de outros mecanismos, como a disfunção serotoninérgica secundária à deficiência de produção ou de aproveitamento dos hormônios tireoideanos ${ }^{(9,20,21)}$.

A FM é reconhecida como um conjunto de sintomas relacionados ao estresse, que por si só, pode acarretar alterações do sistema neuroendócrino envolvendo o eixo hipotálamo-hipófise-adrenal (HHA) e suas interações recíprocas com outras funções endócrinas como a gonadal, tireoideana, do crescimento e principalmente do sistema nervoso autônomo. Uma resposta sustentada ao estresse favorece o aumento do hormônio liberador de corticotropina (CRH), que por sua vez estimula a secreção de somatostatina, a qual inibe a produção de hormônios sexuais e tireoideanos, além do hormônio de crescimento ${ }^{(22,23)}$.

Por não existir pesquisa que comprove os mecanismos fisiopatológicos da FM, os critérios diagnósticos dessa doença devem ser utilizados com cautela. $\mathrm{O}$ médico deve 
estar sempre atento para a sintomatologia apresentada por estes pacientes.

Este trabalho vem ainda confirmar a assertiva dos critérios de classificação para o diagnóstico de FM (ACR 1990 $)^{(3)}$ de que "o conceito de FM primária e secundária não deve existir, pois o curso clínico da fibromialgia é independente e o mesmo, em ambas as situações".

Sintomas presentes na FM, como dor muscular, fadiga, diminuição da capacidade física e intolerância ao frio, são semelhantes aos sintomas presentes em diversas disfunções

\section{REFERÊNCIAS}

1. Haun MVA, Heymann RE, Helfestein M, Feldman D: Especial Fibromialgia. Sinopse de Reumatologia, ano 3, $\mathrm{n}^{\mathrm{O}} 1,4-8,2001$.

2. Moreira C, Carvalho MAP: Noções Práticas de Reumatologia. Editora Health, 1996.

3. Wolfe F et al: The American College of Reumatology 1990, Criteria for the Classification of Fibromialgia. Report of the Multicenter Criteria Commitee. Arthritis Rheum 33: 160-172, 1990.

4. Golding DN: Hypothyroidism presenting with musculo-skeletal symptons. Ann Rheum Dis 29: 10-14, 1970.

5. Neeck G, Riedel W: Thyroid function in pacients with fibromyalgia syndrome. J Rheumatol 19: 1120-1122, 1992.

6. Wilson J, Walton JN: Some muscular manifestation of hypothyroidism. J Neurol Neurosurg Psychiatr 22: 320-324, 1959.

7. Bland JH, Frymoyer JW: Rheumatic syndromes of myxedema. N Engl Med 282: 1171-1174, 1970.

8. Wilke SW, Sheeler LR, Makarowski WS: Hypothyroidism with presenting symptons of fibrositis. J Rheumatol 8: 627-630, 1981.

9. Lowe JC, Cullum ME, Graf JR LH, Yellin J: Mutations in the c-erbAB_gene: do they underlie euthyroid fibromyalgia? Medical Hypotheses 48: 125-135, 1997.

10. Delamere JP, Scott DL, Felix-Davies DD: Thyroid dysfunction endócrinas como o hipotiroidismo, insuficiência adrenal, insuficiência do hormônio de crescimento ${ }^{(24)}$. Por outro lado, doenças da tireóide são comumente encontradas e podem ser prontamente identificadas e controladas. Para uma melhor abordagem diagnóstica e posterior tratamento dos pacientes, há que se considerar a presença de manifestações clínicas ou laboratoriais de disfunção tireoideana nos pacientes portadores de FM, além de FM em pacientes portadores de hipotireoidismo.

and rheumatic diseases. J Roy Soc Med 75: 102, 1982.

11. Forslind K, Fredriksson E, Nived O. Does Primary Fibromyalgia exist? Brit J Rheumatol: 368-70,1990.

12. Shiroky JB, Cohen M, Ballachey ML, Neville C: Thyroid dysfunction in rheumatoid arthritis: A controlled prospective survey. Ann Rheum Dis 52: 454-456, 1993.

13. Lowe JC: Thyroid status of 38 fibromyalgia patients: implications for the etiology of fibromyalgia. Clin Bull Myofascial Ther 2: 36-41, 1996.

14. Ferraccioli G, Cavalieri F, Salaffi F et al: Neuroendocrinologic findings in primary fibromyalgia (soft tissue chronic pain syndrome) and in other chronic rheumatic conditions (rheumatoid arthritis, low back pain). J Rheumatol 17: 869-873, 1990.

15. Moldofsky H, Warsh JJ: Plasma tryptophan and musculo-skeletal pain in non-articular rheumatism. Pain 5: 65-71, 1978.

16. Russel IJ, Michalek JE, Vipraio GA, Fletcher EM, Javors MA, Bowden CA: Platelet $3 \mathrm{H}$-imipramine uptake receptor density and serum serotonin levels in patients with fibromyalgia/fibrositis syndromes. J Rheumatol 19: 104-109, 1992.

17. Houvenagel E: 5-Hydroxy-indoleacetic acid in cerebrospinal fluid in fibromyalgia. Arthritis Rheum 33: 555,1990.

18. Russel IJ, Vaeroy H, Javors M, Nyberg F: Cerebrospinal fluid biogenic amine metabolites in fibromyalgia/fibrositis syndrome and rheumatoid arthritis. Arthritis Rheum 35: 550-556, 1992. 